\title{
Variables Separation in Gravity
}

\section{Valery V. Obukhov*†}

Tomsk State Pedag. Univ., Tomsk, Russia

E-mail: obukhovetspu.edu.ru

\section{Konstantin E. Osetrin}

Tomsk State Pedag. Univ., Tomsk, Russia

E-mail: osetrinetspu.edu.ru

\begin{abstract}
To solve the problem of exact integration of the field equations or equations of motion of matter in curved spacetimes one can use a class of Riemannian metrics for which the simplest equations of motion can be integrated by the complete separation of variables method. Here, we consider the particular case of the class of Stäckel metrics. These metrics admit integration of the HamiltonJacobi equation for test particle by the complete separation of variables method. It appears that the other important equations of motion (Klein-Gordon-Fock, Dirac, Weyl) in curved spacetimes can be integrated by complete separation of variables method only for the metrics, belonging to the class of Stäckel spaces.
\end{abstract}

Fourth International Winter Conference on Mathematical Methods in Physics 09 - 13 August 2004

Centro Brasileiro de Pesquisas Fisicas (CBPF/MCT), Rio de Janeiro, Brazil

\footnotetext{
${ }^{*}$ Speaker.

${ }^{\dagger}$ The author thanks RFBR for financial support (grant N 03-01-00105).
} 


\section{Introduction}

One of the main problems of mathematical physics for the gravity theory is the problem of exact integration of the field equations or equations of motion of matter. To solve this problem one can use the class of Riemannian metrics for which the simplest equations of motion can be integrated by the complete separation of variables method. Apparently in this class the Stäckel metrics are of the same interest. Recall that metric is called the Stäckel one if the Hamilton-Jacobi equation

$$
g^{i j} S_{, i} S_{, j}=m^{2} \quad i, j, k, l=1, \ldots n
$$

can be integrated by the complete separation of variables method. In this case the privileged coordinate set $\left\{u^{i}\right\}$ exists for which complete integral of eq. (1.1) can be shown in the form

$$
S=\sum_{i=1}^{n} \phi_{i}\left(u^{i}, \lambda\right)
$$

where $\lambda_{i}-$ is the essential parameter.

It appears that the other important equations of motion (Klein-Gordon-Fock, Dirac, Weyl) can be integrated by complete separation of variables method only for the metrics, belonging to the class of Stäckel spaces. That is why the research of this class of spaces belongs to the one of the important problems of the mathematical physics. In the present report we consider the following parts of this problem:

- The problem of complete separation of variables for the Hamilton-Jacobi equation.

- Integration of Einstein equations for the Stäckel spaces.

- Conformally Stäckel spaces.

- Homogeneous Stäckel spacetimes.

\section{Stäckel spaces}

The theory of Stäckel spaces has been developed by many authors. Let us recall the main statements and enumerate the main theorems of the Stäckel spaces theory.

Definition 1. Let $V_{n}$ be a n-dimensional Riemannian space with metric tensor $g_{i j}$. The Hamilton - Jacobi equation can be integrated by complete separation of variables method if co-ordinate set $\left\{u^{i}\right\}$ exists for which complete integral can be presented in the form (1.2).

Definition 2. $V_{n}$ is called the Stäckel space if the Hamilton-Jacobi equation (1.1) can be integrated by complete separation of variables method.

The following theorem was proved by V.N. Shapovalov [1]-[2]. 
Theorem 1. Let $V_{n}$ be the Stäckel space. Then $g_{i j}$ in privileged co-ordinate set can be shown in the form

$$
\begin{gathered}
g^{i j}=\left(\Phi^{-1}\right)_{n}^{v} G_{v}^{i j}, \quad G_{v}^{i j}=G_{v}^{i j}\left(u^{v}\right), \quad \Phi_{\mu}^{v}=\Phi_{\mu}^{v}\left(u^{\mu}\right) \\
G_{v}^{i j}=\delta_{v}^{i} \delta_{v}^{j} \varepsilon_{v}\left(u^{v}\right)+\left(\delta_{v}^{i} \delta_{p}^{j}+\delta_{v}^{j} \delta_{p}^{i}\right) G_{v}^{v p}+\delta_{p}^{i} \delta_{q}^{j} G_{v}^{p q}, \quad p, q=1, \ldots N, \quad v, \mu=N+1, \ldots, n .
\end{gathered}
$$

where $\Phi_{\mu}^{v}\left(u^{\mu}\right)$ - is called the Stäckel matrix.

It is assumed that summation is performed over repeated superscripts and subscripts provided the symbol $n s(i, j, \ldots)$ (no summation over the indices given in the brackets) does not occur on the right of the formula. One can show that the geodesic equations of Stäckel spaces admit the first integral that commutes pairwise with respect to the Poisson bracket

$$
\underset{\mu}{X}=\left(\Phi^{-1}\right)_{\mu}^{v} H_{v}, \quad H_{v}=\varepsilon_{v} p_{v}^{2}+2 G_{v}^{v p} p_{p} p_{v}+h_{v}^{p q} p_{p} p_{q}, \quad \underset{p}{Y}=\underset{p}{Y^{i}} p_{i}
$$

Thus for a covariant characterization of Stäckel spaces it is sufficient to find determining properties of the integrals (2.1) in an arbitrary co-ordinate system $\left\{x^{i}\right\}_{n}$. Let us write the functions $X_{v}, Y_{p}$ in the form

$$
\underset{v}{X}=\underset{v}{X}{ }^{i j} p_{i} p_{j}, \quad \underset{p}{Y}=\underset{p}{Y} p_{i}^{i}, \quad \text { then } \quad \underset{v}{X}(i j ; k)=\underset{p}{Y}(i ; j)=0
$$

(the semicolon denotes the covariant derivative and the brackets denote symmetrization). Therefore $Y_{p}{ }^{i}, X_{\mathrm{v}}{ }^{i j}$ are the components of vector and tensor Killing fields respectively.

Definition 3. Pairwais commuting vector $Y_{p}{ }^{i}$, where $p=1, \ldots N$ and tensor $X_{v}{ }^{i j}$, where $v=N+$ $1, \ldots n$ Killing fields form a complete set of the type $\left(N . N_{0}\right)$ if

$$
\begin{aligned}
& B^{p q} \underset{p}{Y_{q}^{i}} \underset{Y^{j}}{ }+B^{v} \underset{v}{X^{i j}}=0 \quad \Longrightarrow \quad B^{p q}=B^{v}=0 \\
& \operatorname{rank} \mid \underset{p}{Y_{q}^{i}} \underset{q}{Y_{i}} \|=N-N_{0} \\
& \underset{v}{X^{i k}}{ }_{\mu}^{X^{j}}{ }_{k}=C_{v \mu}^{p q} \underset{p}{Y_{p}^{i}} \underset{p}{Y^{j}}+C_{v \mu}^{\tau} \underset{\tau}{X^{i j}} \\
& C_{v \mu}^{\tau}=\Phi_{\rho}^{\tau}\left(\Phi^{-1}\right)_{\nu}^{\rho}\left(\Phi^{-1}\right)_{\mu}^{\rho} /\left(\Phi^{-1}\right)_{n}^{\rho} \\
& \underset{v}{X_{p}^{i j}} \underset{j}{Y}=C_{v p}^{q} \underset{q}{Y^{i}}
\end{aligned}
$$

Theorem 2. A necessary and sufficient geometrical criterion of a Stäckel space is the presence of a complete set of the type (N.N. $)$.

In other words the Hamilton - Jacobi equation can be integrated by the complete separation of variables method if and only if the complete set of the first integrals exists. 


\section{Stäckel spaces and field equations of the theories of gravity}

The metrics of the Stäckel spaces can be used for integrating the field equations of General Relativity and other theories of Gravity. Note that such famous solutions as Schwarcshild, Kerr, NUT, Friedman and others belong to the class of Stäckel spaces. Apparently the first papers devoted to the problem of classification of the Stäckel spaces satisfying the Einstein equations were published by B.Carter [3]. Later in our paper [4] it has been found the complete classification of the special Stäckel electrovac spaces. In other words all Stäckel spaces satisfying the Einstein-Maxwell equations for the case when potentials $A_{i}$ admit complete separation of variables for HamiltonJacobi equation (2.8) have been found. In our paper [7] the classification problem has been solved for the case when $A_{i}$ are arbitrary functions and spaces are null (types (N.1)). In our paper [6] all electrovac spacetimes admitting diagonalization and complete separation of variables for the Dirac-Fock-Ivanenko equation were found.

One of the complicated problems of the modern mathematical physics is the integration problem of the Einstein-Dirac equations. Using the Newman-Penrose formalism one can present these equations in the form

$$
\begin{gathered}
R_{i j}-\frac{1}{2} g_{i j} R=4 \pi G T_{i j}, \\
\nabla_{a b^{\prime}} \xi^{a}=m_{0} \eta_{b^{\prime}}, \quad \nabla_{a b^{\prime}} \eta^{a}=m_{0} \xi_{b^{\prime}}
\end{gathered}
$$

where

$$
\begin{gathered}
T_{i j}=Z_{i}^{a} Z_{j}^{b} \sigma_{a}^{A B^{\prime}} \sigma_{b}^{C D^{\prime}} T_{A B^{\prime} C D^{\prime}}, \quad Z_{i}^{a}=\left(l_{i}, n_{i}, m_{i}, \bar{m}_{i}\right) \\
T_{A B^{\prime} C D^{\prime}}=i k\left(\xi_{D^{\prime}} \nabla_{A B^{\prime}} \xi_{C}+\xi_{B} \nabla_{C D^{\prime}} \xi_{A}-\xi_{C} \nabla_{A B^{\prime}} \xi_{D^{\prime}}-\xi_{A} \nabla_{C D^{\prime}} \xi_{B^{\prime}}-\eta_{D^{\prime}} \nabla_{A B^{\prime}} \eta_{C}-\right. \\
\left.\eta_{B} \nabla_{C D^{\prime}} \eta_{A}+\eta_{C} \nabla_{A B^{\prime}} \eta_{D^{\prime}}+\eta_{A} \nabla_{C D^{\prime}} \eta_{B^{\prime}}\right)
\end{gathered}
$$

and $\nabla_{A B}$ - spinor derivative, $\sigma_{a}^{A B^{\prime}}$ - Infeld-Wan der Varden symbols.

Using spaces for which equation (3.1) can be integrated by the complete separation of variables and separated solutions of the Dirac equation one can transform Einstein-Dirac equations to the set of functional equations. The first papers devoted to the classification problem for the EinsteinDirac equations were done by Bagrov, Obukhov, Sakhapov [8]. The Stäckel spaces of type (3.1) for Einstein-Dirac and Einstein-Weyl equations have been studied. Appropriate solutions have been obtained. They contain arbitrary functions depending on null variable only.

The problem of classification of Stäckel spaces for other theories of gravity for the first time was considered in papers [9]-[10]. The following theories have been considered

1. Brans-Dicke theory. The field equations have the form

$$
\begin{gathered}
R_{i j}-\frac{1}{2} g_{i j} R=\frac{8 \pi}{\phi} T_{i j}-\frac{\omega}{\phi^{2}}\left(\phi_{; i} \phi_{; j}-\frac{1}{2} g_{i j} \phi_{; k} \phi^{; k}\right)-\frac{1}{\phi}\left(\phi_{; i j}-g_{i j} \square \phi\right) \\
\square \phi=\frac{8 \pi}{3+2 \omega} T^{i}{ }_{i}, \quad \square=g^{i j} \nabla_{i} \nabla_{j}, \quad \omega=\text { const } .
\end{gathered}
$$

The case of Einstein-Maxwell equations and the metric has type (N.1) has been considered in paper [9]. All appropriate solutions have been obtained. 
2. The problem for the multiscalar-tensor theory for which field equations have the form

$$
\stackrel{*}{R}_{v \mu}=2<\phi_{, \nu} \phi_{, \mu}>+8 \pi G\left(\stackrel{*}{T}_{v \mu}-\frac{1}{2} \stackrel{*}{g}_{v \mu} \stackrel{*}{T}\right), \quad \stackrel{*}{\square} \phi^{A}+\gamma_{B C}^{A} \phi_{, \nu}^{A} \phi_{, \mu}^{B} \stackrel{*}{v}^{\nu \mu}=4 \pi G \gamma^{A B} \frac{\partial \alpha}{\partial \phi^{B}} \stackrel{*}{T}
$$

was considered in paper [10]. Here $g^{*}{ }_{\nu \mu}, R^{*}{ }_{\nu \mu}$ are metric tensor and Ricci tensor of the space which conformal to space-time,

$$
\stackrel{*}{g}_{v \mu}=\alpha^{2} g_{v \mu}, \quad \alpha=\alpha\left(\phi^{A}\right), \quad<\phi_{v} \phi_{\mu}>=\gamma_{A B} \phi_{, v}^{A} \phi_{, \mu}^{B},
$$

where $\phi^{A}$ - are scalar fields, $\gamma_{A B}=\gamma_{A B}\left(\phi^{C}\right)$ can be considered as a metric tensor of the $n$ dimensional space of scalars,

$$
\gamma_{A B}^{C}=\frac{1}{2} \gamma^{C D}\left(\frac{\partial \gamma_{A D}}{\partial \phi^{B}}+\frac{\partial \gamma_{D B}}{\partial \phi^{A}}-\frac{\partial \gamma_{A B}}{\partial \phi^{C}}\right), \quad \stackrel{*}{\square} \phi^{A} \equiv\left(\sqrt{\left|g^{*}\right|} \stackrel{*}{g}^{v \mu} \phi_{, v}^{A}\right)_{, \mu} / \sqrt{|\stackrel{*}{g}|}
$$

3. The classification problem for the Einstein-Vaidya equations. Let the stress-energy tensor have the form

$$
T_{i j}=T_{i j}^{(e)}+a(x) l_{i} l_{j}, \quad l_{i} l^{i}=0
$$

then Einstein equations can by written in the following way

$$
R_{i j}-\frac{1}{2} g_{i j} R=4 \pi G\left(T_{i j}^{(e)}+a l_{i} l_{j}\right)
$$

If $T_{i j}^{(e)}$ has the form for electrovac, and $F_{i j}$ satisfies the Maxwell equations (2.12) the solutions of the equations (3.5) are electrovac ones. For these equations the classification problem was solved in paper [7] for the case when the complete set has type (N.1) (null case). In other words all metrics and electromagnetic potentials satisfying equations (3.5) provided that Hamilton-Jacobi equation (1.1) can be integrated by the complete separation of variables method for null Stäckel spaces have been found.

\section{Conformally Stäckel spaces}

Let us consider the Hamilton-Jacobi equation for a massless particle

$$
g^{i j} S_{, i} S_{, j}=0
$$

Obviously this equation admits complete separation of variables for a Stäckel space. Yet one can verify that if $g^{i j}$ has the form

$$
g_{i j}=\tilde{g}_{i j}(x) \exp 2 \omega(x)
$$

where $\tilde{g}_{i j}$ is a metric tensor of the Stäckel space, then eq.(4.1) can be solved by complete separation of variables method too. In paper [2] it was proved that (4.2) is necessary and additional condition of the complete separation of variables. Note that conformally Stäckel spaces play important role when massless quantum equations are considered (f.e. conformal invariant Chernikov-Penrose equation, Weyl's equation etc.). That is why the problem of investigation of Einstein spaces which admits complete separation of variables in eq.(4.1) is exceptionally interesting. Apparently the 
first attempt to consider this problem was taken in paper [12]. The next step was done in paper [13], where some of metrics belonging to conformal Stäckel spaces of type (N.1) was studied. The problem of classification of conformally Stäckel spaces satisfying the Einstein equation

$$
R_{i j}=\Lambda g_{i j}, \quad \Lambda=\text { const }
$$

is more difficult than appropriate problem for the Stäckel spaces. To obtain the functional equations from eq. (4.3) one has to use the integrability conditions. These conditions was found by Brinkman [11]. Let us denote $V_{n}$ the Riemannian space with metric tensor $g_{\alpha \beta}, \tilde{V}_{n}$ be an Einstein's space with metric tensor $\tilde{g}_{\alpha \beta} . \tilde{R}_{\alpha \beta}, \tilde{R}_{\alpha \beta \gamma \delta}, \tilde{R}$ are components of Ricci tensor, Riemann tensor and scalar curvature respectively for the space $\tilde{V}_{n}$, and $R_{\alpha \beta}, R_{\alpha \beta \gamma \delta}, R$ are those tensors for the space $V_{n}$. Moreover we denote:

$$
T_{\alpha \beta}=\frac{1}{n-2}\left(R_{\alpha \beta}-\frac{R g_{\alpha \beta}}{2(n-1)}\right), \quad W=\frac{1}{2}(\nabla \omega)^{2}-\frac{\Lambda}{2(n-1)} \exp 2 \omega
$$

where $\omega_{; \alpha} \equiv \nabla_{\alpha} \omega$ are the covariant derivatives in $V_{n}$.

We can write (4.3) in the form

$$
\omega_{, \alpha ; \beta}-\omega_{, \alpha} \omega_{, \beta}+W g_{\alpha \beta}+T_{\alpha \beta}=0 .
$$

Brinkman has shown that integrability conditions of eq. (4.4) have the form

$$
\omega_{, \delta} C_{\alpha \beta \gamma}^{\delta}=S_{\alpha \beta \gamma}, \quad \text { where } \quad S_{\alpha \beta \gamma} \equiv T_{\alpha \gamma ; \beta}-T_{\alpha \beta ; \gamma}
$$

where $C_{\alpha \beta \gamma \delta}$ are the components of Weyl tensor. In our paper [13] eqs.(4.5) have been presented in a more simple form. To simplify them we use the consequence from Bianchi identities

$$
R_{\alpha \beta \gamma ; \sigma}^{\sigma}=R_{\alpha \beta ; \gamma}-R_{\alpha \gamma ; \beta} .
$$

One can take out the following correlation

$$
C_{\beta \gamma \delta ; \alpha}^{\alpha}=R_{\beta \gamma \delta ; \alpha}^{\alpha}+S_{\beta \gamma \delta}-\frac{1}{2(n-1)}\left(g_{\beta \gamma} R_{, \delta}-g_{\beta \delta} R_{, \gamma}\right) .
$$

That is why

$$
C_{\alpha \beta \gamma ; \delta}^{\delta}=-(n-3) S_{\alpha \beta \gamma}
$$

and Brinkman's conditions can be presented in the form

$$
\omega_{, \delta} C_{\alpha \beta \gamma}^{\delta}=-\frac{1}{(n-3)} C_{\alpha \beta \gamma ; \delta}^{\delta},
$$

finally

$$
\nabla_{\delta}\left(C_{\alpha \beta \gamma}^{\delta} \exp (n-3) \omega\right)=0 .
$$

Using (4.9) we have proved the following theorem [13]

Theorem 3. Let $g_{i j}$ be the metric tensor of the Stäckel space of type (N.1). Then Einstein space conformal to $\tilde{V}_{4}$ admits the same Killing vectors as $V_{4}$.

Moreover one can prove the following statement.

Theorem 4. Let $\tilde{V}_{n}$ is conformally Stäckel space of type (N.1) $(N \geq 2)$ satisfying the Einstein equation (4.3). Then Hamilton-Jacobi equation (1.1) admits the complete separation of variables.

In other words all null conformally Stäckel Einstein spaces belong to the class of the null ones. Nontrivial null conformally Stäckel solutions of the Einstein equations belong only to (1.1)-type. 


\section{Homogeneous Stäckel spacetimes}

One of the problems of cosmology is the problem of finding exact realistic models. The most interesting for cosmology are space-homogeneous models, which are known to admit a 3parametrical transitive group of motions with space-like orbits.

On the other hand, it is known that geodesic equations in the form of Hamilton-Jacobi can be integrated by the complete separation of variables method if they admit a first integrals which are linear and quadratic with respect to the momenta $Y_{p}=Y_{p}{ }^{i} p_{i}, X_{v}=X_{v}{ }^{i j} p_{i} p_{j}$, where $Y^{i}$ - Killing vectors and $X^{i j}$ - Killing tensors.

Thus there is a problem of finding a subclass of homogeneous space-times admitting complete sets of integrals of motion. In other words, a space-time with a complete set must admit a 3parametrical transitive group of motions with space-like orbits.

There are 7 types of complete sets for space-times with the signature $(-,+,+,+)$. In our papers [14]-[15] we find a complete classification of homogeneous Stäckel space-times (3.1) type.

Let us consider space-times with a complete set of type (2.1). The contravariant metric tensor of the Stäckel space of type (2.1) in a privileged coordinate system can be written as (signature $(-,+,+,+))$

$$
g^{i j}=\frac{1}{\Delta}\left(\begin{array}{cccc}
1 & 0 & 0 & 0 \\
0 & 0 & f\left(x^{1}\right) & 1 \\
0 & f\left(x^{1}\right) & c\left(x^{0}, x^{1}\right) & b\left(x^{0}\right) \\
0 & 1 & b\left(x^{0}\right) & a\left(x^{0}\right)
\end{array}\right)
$$

where $\Delta=d_{0}\left(x^{0}\right)+d_{1}\left(x^{1}\right), \quad c=c_{0}\left(x^{0}\right)+c_{1}\left(x^{1}\right)$

$$
\operatorname{det} g^{i j}=-\frac{D}{\Delta^{4}}, \quad D=a f^{2}-2 b f+c>0
$$

The complete set of type (2.1) includes the following Killing vectors:

$$
X_{1}=(0,0,0,1), \quad X_{2}=(0,0,1,0)
$$

The vector $X_{2}$ is space-like because $D>0$; however, the restriction of the metric to the orbits of subgroup $X_{1}, X_{2}$ is degenerated. It is a general property of Stäckel spaces with $N_{0} \neq 0$, which are called null spaces. The breaking of space-likeness of the orbits of the complete set subgroup demands the introduction of an additional Killing vectors $-X_{3}$ and $X_{4}$.

The commutation relations are

$$
\begin{aligned}
& {\left[X_{1}, X_{2}\right]=0} \\
& {\left[X_{1}, X_{a}\right]=\alpha_{a} X_{1}+\beta_{a}{ }^{b} X_{b}, \quad a, b=3,4} \\
& {\left[X_{2}, X_{a}\right]=\gamma_{a}{ }^{2} X_{2}+\gamma_{a}^{b} X_{b}} \\
& {\left[X_{3}, X_{4}\right]=\gamma_{5} X_{3}+\gamma_{6} X_{4}+\gamma_{7} X_{2}}
\end{aligned}
$$

To find algebraic classes, the vectors of this subgroup can be subjected to the linear transformation

$$
\tilde{X}_{\alpha}=S_{\alpha}{ }^{\beta} X_{\beta}, \quad \alpha, \beta=2 . .4
$$

Also, one can use a coordinate transformation keep the form of the metric tensor

$$
\begin{array}{lrl}
\tilde{x^{p}} & =\alpha^{p} x^{p} & p, q=0,1 \\
\tilde{x^{v}}=\alpha^{v}+\beta_{\mu}{ }^{v}\left(x^{p}\right) x^{\mu} & \mu, v=2,3
\end{array}
$$


Applying the admissible transformations and the Jacobi identities to the commutation relations, integrating them and Killing equations we obtain complete classification of this space-times (all metrics and Killing vectors) and establish the following result.

Theorem 5. Homogeneous Stäckel space of type (2.1) have only Petrov's type III or type N metrics with constant scalar curvature. This spacetimes can not be Bianchi type VIII or IX.

This work partially supported by RFBR, grant N 03-01-00105.

\section{References}

[1] V.N. Shapovalov, The Stäckel spaces, Sib. Math. Journal (Sov. Journ. of Math.) 20 (1979) 1117.

[2] V.N. Shapovalov, Symmetry and separation of variables in Hamilton-Jacobi equation, Izv. vuzov. Fizika (Sov. Phys. Journ.) 9 (1978) 18.

[3] B. Carter, Hamilton-Jacobi and Schrodinger separable solutions of Einsteins equations, Comm. Math. Phys. 10 (1968) 280.

[4] V.G. Bagrov, V.V. Obukhov, A.V. Shapovalov, Special Stäckel electrovac space times, Pramana J. Phys. 26 (1986) 93.

[5] V.G. Bagrov, V.V. Obukhov, Separation of variables for the Klein-Gordon equation in special Stäckel space-times, Class. Quant. Grav. 7 (1990) 19.

[6] V.G. Bagrov, V.V. Obukhov, New method of integration for the Dirac equation on a curved space-time, J. Math. Phys. 33 (1992) 2279.

[7] V.G. Bagrov, V.V. Obukhov, K.E. Osetrin, Classification of null-Stäckel electrovac metrics with cosmological constant, Gen. Rel. Grav. 20 (1988) 1141.

[8] V.G. Bagrov, V.V. Obukhov, A.G. Sakhapov, Integration of the Einstein-Dirac equations, J. Math. Phys. 37 (1996) 5599.

[9] V.G. Bagrov, V.V. Obukhov, Exact solutions of vacuum Brans-Dicke equations, Izv. Vuzov. Fizika (Sov. Phys. Journ.) 1 (1992) 86.

[10] V.G. Bagrov, V.V. Obukhov, Exact integration of scalar equations in multiscalar - tensor theory, Izv. Vuzov. Fizika (Russ. Phys. Journ.) 2 (1992) 79.

[11] H.W. Brinkman, Riemann spaces conformal to Einstein's spaces, Ann. Math. 91 (1994).

[12] V.V. Obukhov, Classes of exact solutions of Einstein equation, Kandidat. Dissertat, Moscow State University, Moscow 1979.

[13] V.G. Bagrov, V.V. Obukhov, K.E. Osetrin, Tetrade form of Brinkman's conditions, Proc. II Alexander Friedmann Inter. Seminar on Grav. \& Cosmol. Publ. Central Astronomical Observatory, St.-Petersburg 1994.

[14] V.G. Bagrov, V.V. Obukhov, K.E. Osetrin, A.E. Filippov, Stäckel spacetimes with additional symmetries, Grav. \& Cosmol. 5 (1990) 10.

[15] V.V. Obukhov, K.E. Osetrin, A.E. Filippov, Metrics of homogeneous spacetimes with complete sets of the (3.1) type, Izv. Vuzov Fizika (Russ. Phys. Journ.) 1 (2002) 42. 